\title{
Abnormal iron metabolism in fibroblasts from a patient with the neurodegenerative disease hereditary ferritinopathy
}

\author{
Ana G Barbeito ${ }^{1}$, Thierry Levade², Marie B Delisle ${ }^{3}$, Bernardino Ghetti ${ }^{1}$, Ruben Vidal ${ }^{{ }^{*}}$
}

\begin{abstract}
Background: Nucleotide duplications in exon 4 of the ferritin light polypeptide (FTL) gene cause the autosomal dominant neurodegenerative disease neuroferritinopathy or hereditary ferritinopathy (HF). Pathologic examination of patients with HF has shown abnormal ferritin and iron accumulation in neurons and glia in the central nervous system (CNS) as well as in cells of other organ systems, including skin fibroblasts. To gain some understanding on the molecular basis of HF, we characterized iron metabolism in primary cultures of human skin fibroblasts from an individual with the FTL C.497_498dupTC mutation.

Results: Compared to normal controls, HF fibroblasts showed abnormal iron metabolism consisting of increased levels of ferritin polypeptides, divalent metal transporter 1, basal iron content and reactive oxygen species, and decreased levels of transferrin receptor-1 and IRE-IRP binding activity.

Conclusions: Our data indicates that HF fibroblasts replicate the abnormal iron metabolism observed in the CNS of patients with HF. We propose that HF fibroblasts are a unique cellular model in which to study the role of abnormal iron metabolism in the pathogenesis of HF without artifacts derived from over-expression or lack of endogenous translational regulatory elements.
\end{abstract}

\section{Background}

Abnormal brain iron metabolism leading to neurodegeneration is the main feature of diseases such as Friedreich ataxia (FRDA), aceruloplasminemia, neurodegeneration with brain iron accumulation type I (NBIA I), and hereditary ferritinopathy (HF) or neuroferritinopathy [1-3]. HF is an adult-onset autosomal dominant disease caused by nucleotide duplications in exon 4 of the ferritin light polypeptide $(F T L)$ gene. Six different mutations have been reported, leading to an increase in the length and a change of the amino acid sequence of the C-terminus of FTL [4-9]. HF affects the central nervous system (CNS) presenting clinically as an extra-pyramidal movement disorder accompanied by cognitive and behavioral disturbances, starting between the third and sixth decade of life [10]. Neuropathologically, HF is characterized

\footnotetext{
* Correspondence: rvidal@iupui.edu

'Department of Pathology and Laboratory Medicine and Indiana Alzheimer disease Center, Indiana University School of Medicine, 635 Barnhill Dr, MSB A136, Indianapolis, IN, 46202, USA

Full list of author information is available at the end of the article
}

by a severe neuronal loss in the basal ganglia, atrophy of cerebellum and cerebral cortex, abnormal iron accumulation, and the presence of ferritin inclusion bodies (IBs) in neurons and glia [3]. Ferritin IBs are not limited to the CNS since they can also be seen in hepatocytes, cells of the renal tubular epithelium, endothelial cells of capillaries, and skin fibroblasts $[5,6]$.

Ferritin is the main intracellular iron storage protein, having a central role in the regulation of cellular iron metabolism and iron detoxification [11,12]. Mammalian ferritin consists of 24 subunits of FTLs and ferritin heavy polypeptides (FTH); the FTH subunit is involved in the rapid detoxification of iron, whereas the FTL subunit facilitates iron nucleation, mineralization, and long-term iron storage [13]. Ferritin provides both a source of metabolic active iron and also serves as an oxygen free radical cytoprotective protein, storing iron that is not needed for immediate metabolic use $[11,12]$. Each subunit consists of a bundle of 4 parallel $\alpha$-helices (A, B, C, and D), a long extended loop (connecting helices $B$ and $C$ ), and a $C$-terminus with a short $\alpha$-helix 
(E) which is involved in important stabilizing interactions around the 4-fold symmetry axes [12]. Spectroscopic and biochemical studies of recombinant mutant FTL homopolymers assembled from the p. Phe167SerfsX26 polypeptide (originated from the c.497_498dupTC mutation) [5] have shown that the mutation causes conformational changes in ferritin, altering iron incorporation and promoting ironmediated aggregation of ferritin. The process of ironinduced aggregation of ferritin does not seem to involve covalent bonds since it can be reversed by iron-chelants both in vitro and in vivo [14]. X-ray crystallographic analysis of homopolymers of the mutant p.Phe167SerfsX26 polypeptide showed the complete absence of the E helical domain of FTL in mutant subunits and substantial disruption of the 4-fold pores of the 24-mer [15]. Transgenic expression of the p.Phe167SerfsX26 polypeptide in mice recapitulated several features of the disease, including intracellular formation of ferritin IBs in neurons and glia in the CNS and in cells of other organ systems, including skin fibroblasts [16]. Transgenic mice showed dysregulation of iron homeostasis and evidence of oxidative damage in the brain, similarly to what has been observed in individuals with HF [17]. Herein, we report ferritin accumulation, iron dyshomeostasis and evidence of oxidative stress in human skin fibroblasts from a patient with HF. Our results reveal that the broad dysfunction of iron homeostasis observed in individuals with HF and in the transgenic animal model of HF is replicated in HF skin fibroblasts. We propose that HF skin fibroblasts represent a unique cellular model in which to study the molecular mechanisms of cellular toxicity that may lead to neurodegeneration in HF.

\section{Results}

Ferritin polypeptides accumulate in skin fibroblasts expressing mutant FTL

Confocal fluorescence microscopy analysis (Figure 1) using abs against the mutant-FTL chain showed that the mutant polypeptide accumulated both in the cytoplasm and in the nucleus of HF fibroblasts (Figure 1e), as previously reported in skin fibroblasts of individuals with HF and transgenic mice $[5,16]$. No staining was observed in normal control skin fibroblasts (non-HF) (Figure 1b). Western blot analysis showed expression of the ferritin subunits in normal and in HF fibroblasts (Figure 2a). Antibodies against the $\mathrm{N}$-terminus of FTL detected two bands on western blots of HF fibroblasts, in agreement with the difference in molecular weight between wild-type and mutant-FTL polypeptides [5]. Densitometric analysis showed a statistically significant $(\mathrm{p}<0.05)$ increase in the levels of ferritin light and heavy polypeptides in HF fibroblasts (FTH1: 3.2 fold; FTL: 4.7 fold) (Figure 2b).

\section{Altered expression of proteins of iron metabolism in HF fibroblasts}

Western blot analysis of the expression of TfR 1 and DMT1 + and - IRE isoforms (+/-IRE-DMT1) showed significant changes in the levels of both proteins (Figure 3a, b). Densitometric analysis showed a statistically significant ( $\mathrm{p}<0.05$ ) decrease in the levels of TfR1 (reaching a level of $70 \pm 11 \%$ of non-HF control) and a statistically significant $(\mathrm{p}<0.05)$ increase in the total levels of the iron influx transporter +/-IRE-DMT1 (118 $\pm 10 \%$ of non-HF control), mainly in bands corresponding to molecular weights of $\sim 70 \mathrm{kDa}$ and $\sim 50 \mathrm{kDa}$. No significant changes were observed for FPN (Figure 3c, f).

\section{Cellular iron status in HF fibroblasts}

Total iron contents were measured using a ferrozinebased iron assay [18]. Mutant fibroblasts showed a statistically significant $(\mathrm{p}<0.05)$ increase in the levels of basal iron content when compared to non-HF fibroblasts (non-HF: $10.9 \pm 1.2 \mathrm{nmol} \mathrm{Fe} / \mathrm{mg}$ protein; HF: 13.6 $\pm 1.4 \mathrm{nmol} \mathrm{Fe} / \mathrm{mg}$ protein) (Figure $4 \mathrm{a}$ ). LIP, the iron available for metabolism processes in the cell, was measured using a modified calcein assay [19-21]. No statistically significant differences in the levels of LIP were observed under basal conditions (BC) between HF and non-HF fibroblasts. LIP levels in normal and HF fibroblasts increased with statistical significance upon exposure to $100 \mu \mathrm{M}$ FAC (Figure $4 \mathrm{~b}$ ).

\section{IRE-IRP binding activity}

Under basal conditions, IRE-IRP binding was found to be reduced in HF fibroblasts when compared to normal fibroblasts (non-HF: $100 \pm 12 \%$; HF: $88 \pm 8 \%$ ) (Figure 5). When fibroblasts where subjected to FAC treatment for $72 \mathrm{~h}$, a significant decrease in IRE-IRP binding was observed in HF and non-HF fibroblasts. Interestingly, the change in IRE-IRP binding after treatment with FAC was more pronounced in HF fibroblasts than in control fibroblasts (non-HF: $60 \pm 7 \%$; HF: $24 \pm 9 \%$ ) (Figure 5b). After 2-ME treatment to promote maximal IRE-IRP1 binding $[22,23]$, IRP binding activity increased nearly to $100 \%$, suggesting that the majority of the observed decreased in binding activity may be associated with binding of IRP1 rather than IRP2 [22,23].

\section{Oxidative damage in HF fibroblasts}

ROS formation in cultured fibroblasts was determined using the fluorescent dye 2',7'-dichlorodihydrofluorescein under basal conditions and after treatment with FAC. Under basal conditions, HF fibroblasts showed significant higher levels of ROS than non-HF fibroblasts (Figure 6). A statistically significant increase in cellular oxidant levels was observed in normal and HF fibroblasts after loading with $\mathrm{FAC}$ for $72 \mathrm{~h}$. Interestingly, 


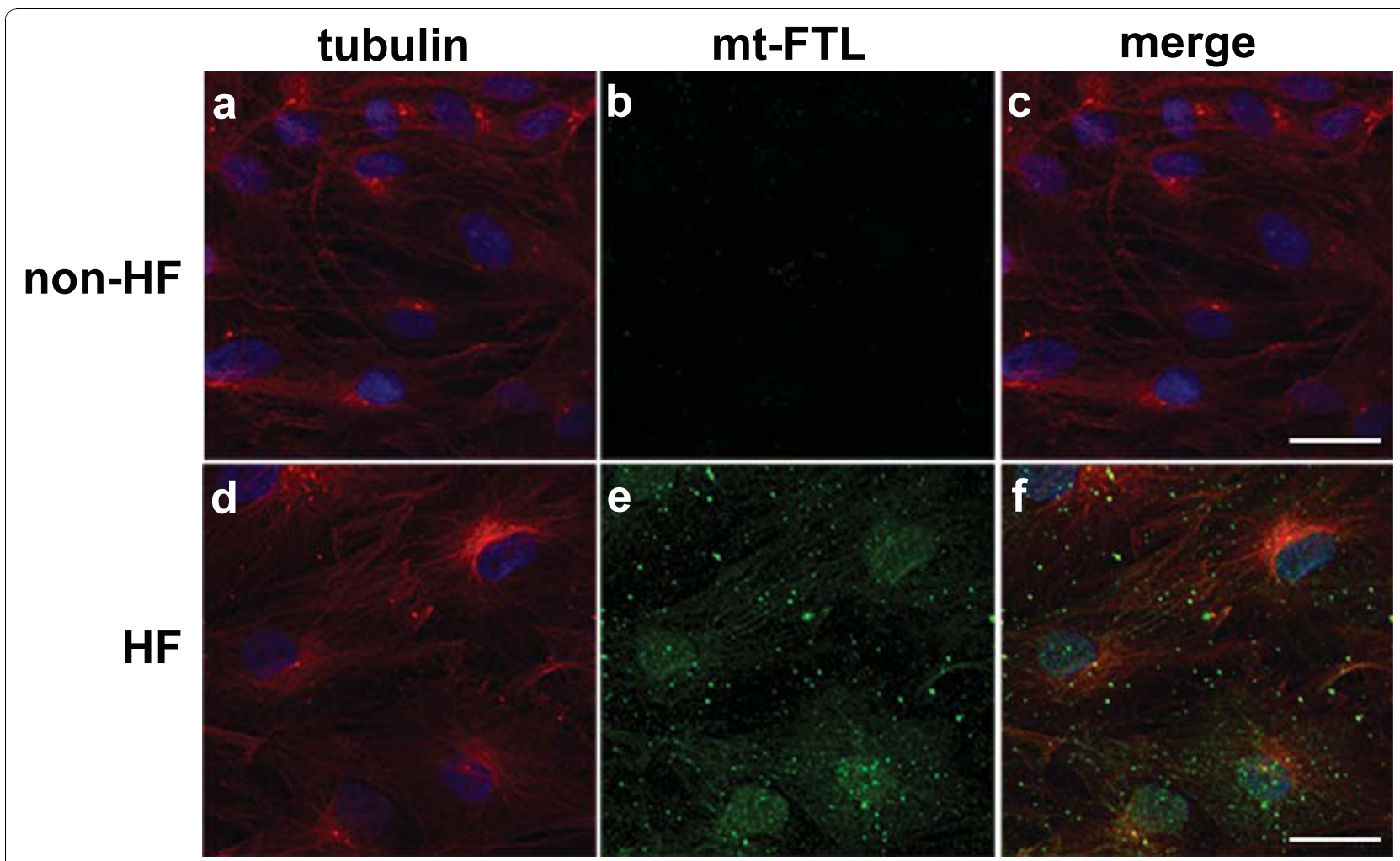

Figure 1 Accumulation of ferritin in cultured HF skin fibroblasts. Confocal immunofluorescence microscopy was performed on cultured confluent wild-type (non-HF) (a-c) and HF (d-f) fibroblasts using antibodies against alpha-tubulin $(a, d)$ and mutant FTL (b, e). Nuclei were stained by DAPI (blue). A merge of the alpha-tubulin (red) and mutant FTL (green) images is shown in c and f. Mutant FTL was observed only in HF fibroblasts, both in the cytoplasm and nucleus. Scale bars: $30 \mu \mathrm{m}$.

ROS levels were significantly higher in HF fibroblasts compared to non-HF fibroblasts after FAC treatment.

\section{Discussion}

The neurodegenerative disease neuroferritinopathy or HF is an autosomal dominant, adult onset disease caused by mutations in the FTL gene that lead to the production of FTL polypeptides with abnormal C-termini [3-9,24]. The clinical presentation of HF varies both within and between families. Patients may present with tremor, cerebellar signs, parkinsonism, psychiatric problems, abnormal involuntary movements (dystonia, chorea), pyramidal syndrome, pseudo-bulbar symptoms, and cognitive deficit [3-10]. Magnetic resonance imaging shows abnormal signals in the globus pallidus and putamen, and cavitation in the putamen $[5,10]$. Mild cerebral and cerebellar atrophy may be observed. Serum ferritin levels may be within normal range or even decreased in some patients [4]. Neuropathologic studies show cavitation in the putamen [4-6]. The cerebrum and cerebellum are atrophic. Ferritin IBs are found in nuclei and cytoplasm of glial cells and neurons in the CNS as well as in cells of other organ systems and are labeled by antibodies against light and heavy chains of ferritin and antibodies specific for the mutant FTL polypeptide [5]. Abnormal iron accumulation (both as ferrous and ferric iron) associated with ferritin IBs has been described [4-6] as well as evidence of oxidative damage in patients [6] and in animal models $[17,25]$. Thus, disrupted iron homeostasis and ironmediated oxidative stress may have a major role in the pathogenesis of HF [3,24].

We investigated cellular iron metabolism in primary cell cultures of skin fibroblasts from an individual with the c.497_498dupTC mutation to determine whether fibroblasts would be an accessible in vitro system in which to directly examine disease-relevant pathologic mechanisms in HF. Our results clearly show alterations of iron metabolism in HF fibroblasts, which seem to recapitulate the abnormalities seen in HF patients and transgenic mice $[5,6,16,17]$. Ferritin is considered a cytoplasmic ironstorage protein; however, a nuclear localization for ferritin [26] has been described in animal models of iron overload [27], and in avian [28] and human astrocytoma cells [29]. In HF fibroblasts, we observed ferritin accumulation in the nucleus and cytoplasm of the cells under basal conditions, as reported in cells from patients affected by HF [5] and in transgenic mice over-expressing mutant FTL [16]. Ferritin accumulation may be explained by the formation of 


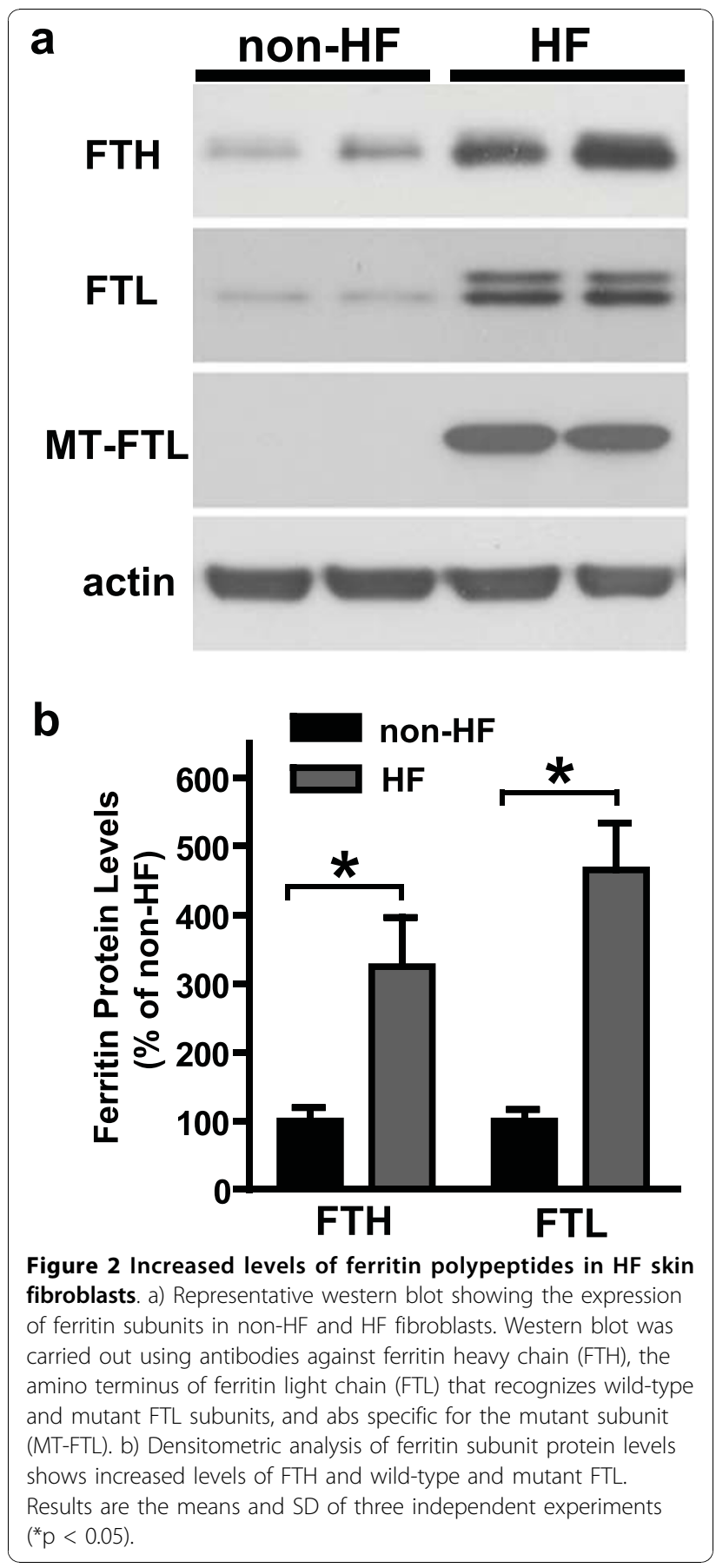

ferritin aggregates and/or by overproduction of ferritin (enhanced transcription/translation of ferritin mRNAs) by the cells in response to a diminished iron-binding activity of mutation-containing ferritin. We have previously shown that ferritin containing the mutant FTL polypeptide has a diminished ability to sequester and store mineralized iron in vitro $[14,15,30]$ and does not lead to an increase in the transcription of ferritin polypeptide genes in vivo in mice, although transgenic mice accumulate ferritin as intracytoplasmic and intranuclear IBs $[16,17]$. Thus, we proposed that the mutant FTL polypeptide may act in vivo as a dominant negative mutant, causing the failure of ferritin in its iron storage function and leading to an increase in the levels of intracellular iron and the translation of ferritin mRNAs (Figure 7) [3]. In HF fibroblasts, we observed an increase in protein levels of ferritin polypeptides and a decrease in the protein levels of TfR1. Proteins of iron metabolism are known to be regulated in a coordinated fashion mainly at the post-transcriptional level [31]. IRPs when interacting with IRE at the 5' -untranslated regions (UTR) of FTH1 and FTL mRNA inhibit their translation, whereas the interaction between IRPs with the IREs located at the 3' UTR of TfR 1 mRNA stabilizes the mRNA, increasing TfR1 protein levels [32]. Thus, the finding of increased protein levels of FTL and FTH and a decrease in TfR1 protein levels in concert with a decrease in the binding activity of IRP-IRE in HF fibroblasts strongly supports the notion of a failure of ferritin in its iron storage function in HF fibroblasts, leading to an increase in FTL and FTH1 translation and degradation of TfR1 mRNA. As would be expected, treatment with FAC of normal and HF fibroblasts leads to a significant decrease in IRE-IRP binding in both cases. However, the change in IRE-IRP binding after treatment with FAC was more pronounced in HF fibroblasts than in control fibroblasts, suggesting a lower IRE-IRP binding activity and consequent translation of ferritin mRNA and destabilization of TfR1 mRNA in HF fibroblasts. In agreement with the data obtained in transgenic mice, no significant changes in the protein levels of the iron exporter FPN were observed [17]. We detected an increase in the protein levels of DMT1 (+/-IRE) in HF fibroblasts, which may account for the increase in total iron observed in HF fibroblasts. DMT1, a proton-coupled metal transporter that participates in iron influx to the cell from the extracellular matrix and/or from recycling endosomes [33] has been shown to contribute to the neurodegenerative process occurring in Parkinson disease (PD), where an increase in the expression of DMT1 (+IRE) in the sustancia nigra of patients with PD correlates with an increase in iron content [34].

The expression profile of iron related-proteins and the IRE-IRP binding activity in HF fibroblasts suggest that the cells might be exposed to elevated levels of intracellular iron. Indeed, we observed that the total cellular iron levels were increased in HF fibroblasts compared to wild-type fibroblasts under basal conditions, although no statistically significant differences between control and HF fibroblast were observed in the levels of the iron available for metabolic processes in the cell (or LIP) under basal conditions. LIP in quiescent conditions represents only a minor fraction of the total cell iron and can undergo dynamic changes in response to 


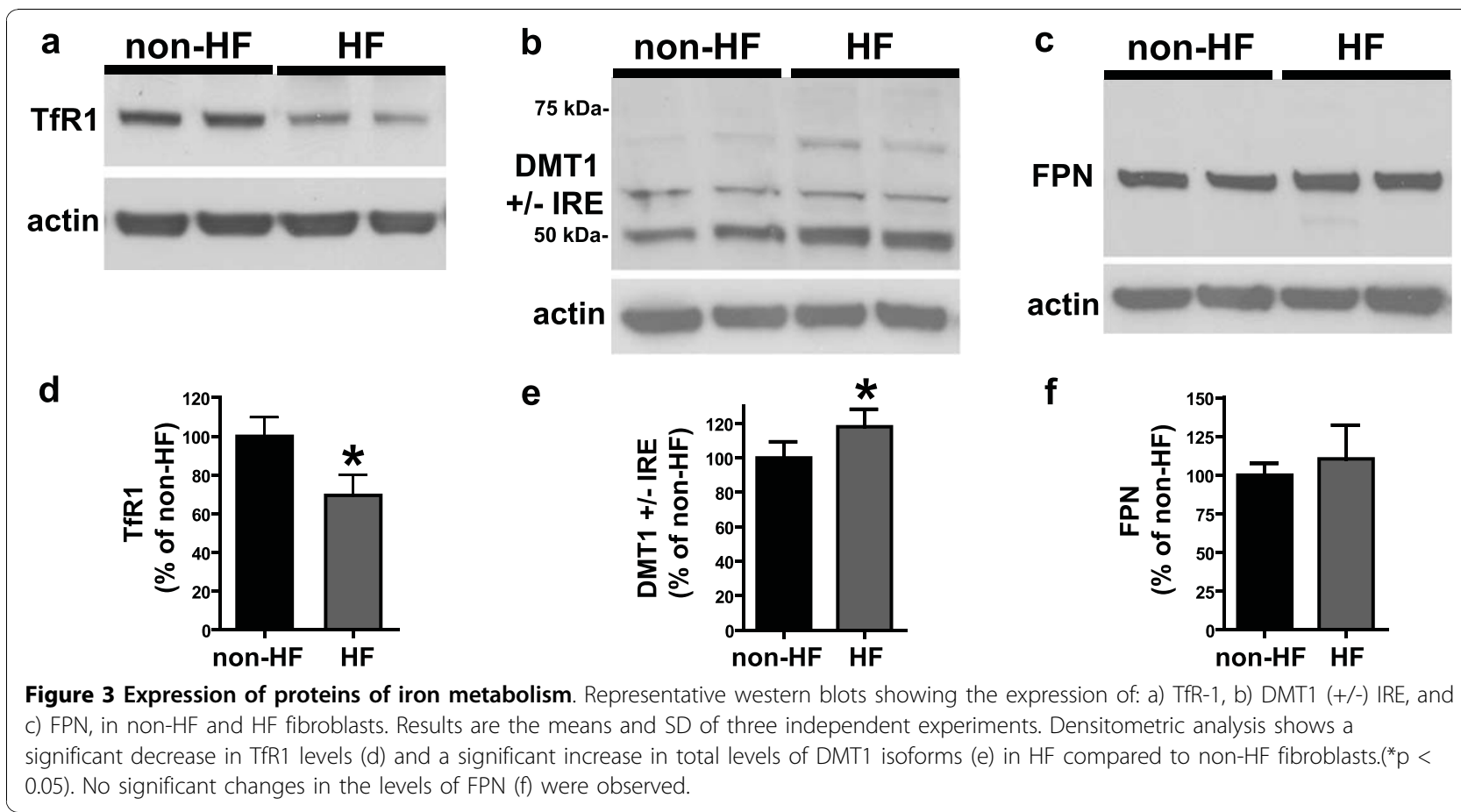

various treatments [35], as seen when $\mathrm{HF}$ and non-HF fibroblasts were challenged with iron. The observed response of LIP to iron treatment suggests differential management of free intracellular iron in HF fibroblasts. HF fibroblasts showed increased ROS production compared to non-HF fibroblasts, which is in agreement with the finding of an increase in iron levels in HF fibroblasts under basal conditions. Iron imbalance and augmented cellular sensitivity to oxidative damage has also been observed in HeLa cells over-expressing the mutant ferritin c.460dupA or the c.497_498dupTC [36]. These data are consistent with studies in transgenic mice, which showed increased iron levels, lipid peroxidation, protein carbonylation, and nitrone products in the CNS [17], and the biochemical and immunohistochemical evidence for oxidative damage, lipid peroxidation and abnormal protein nitration observed in individuals with HF [6].

\section{Conclusions}

The abnormally high iron levels observed in several neurodegenerative disorders have been proposed to lead to oxidative stress and neurodegeneration [17,36-39]. In HF, we found that a defect in a protein of iron metabolism leads to neurodegeneration, directly connecting abnormal iron metabolism and neurodegeneration (Figure 7). Our findings suggest that HF fibroblasts recapitulate the abnormal iron metabolism seen in patients with HF and in a transgenic animal model of HF. Further studies using the HF fibroblast cell model will provide additional insights into the mechanism(s) by which dysfunction of iron homeostasis may lead to neurodegeneration. In addition, they may be important in the characterization of therapeutic agents for HF and related diseases.

\section{Methods}

Materials

Dulbecco's Minimal Essential Medium (DMEM) $4.5 \mathrm{~g} / \mathrm{L}$ glucose, fetal bovine serum (FBS), L-glutamine, penicillin/streptomycin, trypsin-EDTA solution and phosphate buffered saline (PBS) were obtained from Invitrogen (Carlsbad, CA). All cell culture plastics were obtained from Nunc (Rochester, NY, USA). All other general laboratory chemicals were obtained from Sigma-Aldrich Corp. (St. Louis, MO), unless specified.

\section{Fibroblast Cell Culture}

Adult skin fibroblasts were isolated from an individual with the c.497_498dupTC mutation, which has been reported in members of a French family with HF [5]. Normal adult human skin fibroblasts were purchased from PromoCell GmbH (Heidelberg, Germany). Fibroblasts were cultured in DMEM supplemented with $10 \%$ FBS, $2.0 \mathrm{mM}$ L-glutamine, and $100 \mathrm{U} / \mathrm{ml}$ penicillin $/ 100$ $\mu \mathrm{g} / \mathrm{ml}$ streptomycin, and incubated at $37^{\circ} \mathrm{C}$ in humidified air with $5 \% \mathrm{CO}_{2}$. Cells from trypsinized monolayers were seeded at $8-10 \times 10^{3}$ cells $/ \mathrm{cm}^{2}$ and grown to confluence. For treatment with ferric ammonium citrate (FAC), confluent cells were incubated for $72 \mathrm{~h}$ with 100 $\mu \mathrm{M} F A C$ in DMEM in the absence of serum. 


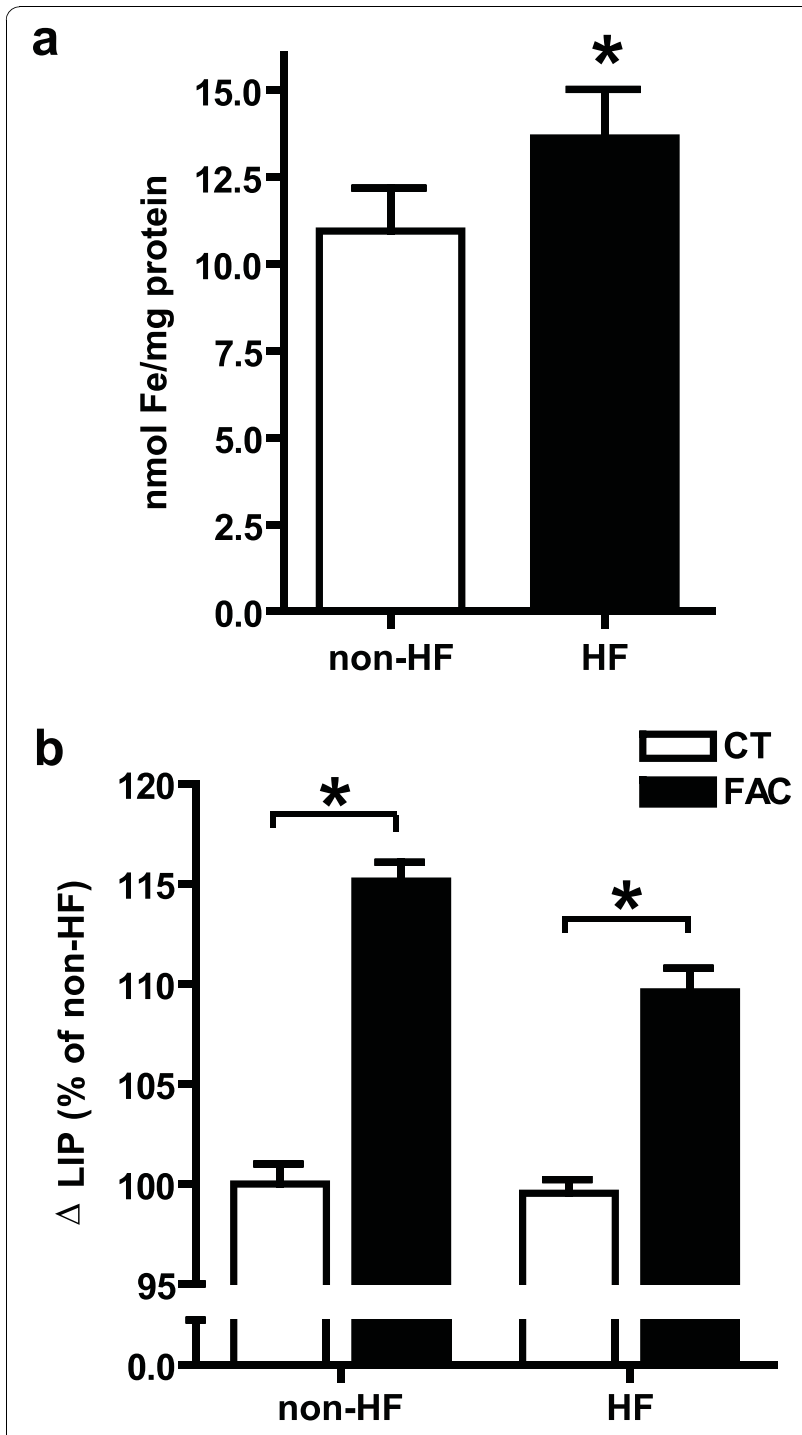

Figure 4 Cellular iron levels. a) Fibroblasts were cultured under basal conditions (BC) and total iron content was analyzed by the ferrozine method. A statistically significant difference $\left({ }^{*} p<0.05\right)$ in the levels of iron content ( $\mathrm{nmol} \mathrm{Fe} / \mathrm{mg}$ protein) was observed in HF compared to non-HF fibroblasts. b) Analysis of the labile iron pool (LIP) in basal conditions and after treatment with $100 \mu \mathrm{M}$ FAC for $72 \mathrm{~h}$. The LIP was measured using the metal-sensitive fluorescence probe calcein. No statistically significant differences were observed under basal conditions. After FAC treatment, LIP levels increased significantly in both non-HF and HF fibroblasts. However, FACinduced levels of LIP were significantly lower in HF compared to non-HF fibroblasts. ${ }^{*} p<0.05$

\section{Western blot analysis}

Confluent cell cultures were lysed in tris buffered saline (TBS) containing 1\% sodium dodecyl sulfate (SDS) and protease inhibitors (PI, Complete, Roche, Indianapolis, IN). Protein extracts $(30 \mu \mathrm{g})$ were run in $4-20 \%$ SDSpolyacrylamide gels (Pierce, Rockford, IL, USA) and transferred to nitrocellulose membranes (GE Healthcare,
Piscataway, NJ, USA). Membranes were blocked for $1 \mathrm{~h}$ in TBS containing $0.1 \%$ Tween-20 and 5\% non-fat dry milk, followed by an overnight incubation with primary antibody (ab). After washing in TBS containing $0.1 \%$ Tween-20, the membranes were incubated with peroxidase-conjugated secondary ab (1:5000; GE Healthcare) for $1 \mathrm{~h}$. Membranes were developed using the ECL chemiluminescent detection system (GE Healthcare). Densitometric analyses were performed using the NIH ImageJ program and normalized against the signal obtained by re-probing the membranes with anti- $\beta$-actin abs (SigmaAldrich). Primary antibodies were: anti-Transferrin Receptor-1 (TfR1; 1:500; Zymed Laboratories, South San Francisco, CA), anti-divalent metal transporter-1 (DMT1, recognizing both +/- IRE isoforms; 1:250; Alpha Diagnostic International, San Antonio, TX), anti-ferroportin-1 (FPN; 1:250; Alpha Diagnostic International), anti-FTL (D-18, 1:250, Santa Cruz Biotechnology, Santa Cruz, CA), anti-mutant FTL (ab1283, 1:8000) that recognizes specifically the human mutant FTL chain [5], anti-FTH (1:2000; Abcam, Cambridge, MA), and anti $\beta$-actin (1:10000; Sigma, St. Louis, MO). Nomenclature of the proteins is according to http:// BioIron.org.

\section{Immunocytochemistry}

Cultured fibroblasts were fixed in PBS $+4 \%$ paraformaldehyde $(\mathrm{pH} 7.4)$, at $4^{\circ} \mathrm{C}$ for $15 \mathrm{~min}$ and washed three times with PBS. Cells were permeabilized and blocked in $10 \%$ goat serum (Invitrogen), $2 \%$ bovine serum albumin (BSA) and $0.1 \%$ Triton X-100 in PBS and incubated with ab1283 (1:100) and mouse anti- $\alpha$ tubulin (1:500; Sigma, St. Louis, MO). Secondary abs were: Alexa-Fluor488 goat anti-rabbit and AlexaFluor594 goat anti-mouse (Molecular Probes Inc, Eugene, OR). Fluorescence images were acquired with a Zeiss LSM 510 confocal microscope using an inverted $40 \times$ NA 1.2 objective.

\section{Electromobility shift assay}

Iron response element (IRE)-binding activity was analyzed by gel retardation assay as described [22,23]. Briefly, cultured confluent fibroblasts were trypsinized and homogenized in extraction buffer (10 mM Hepes, $\mathrm{pH}$ 7.5, $3 \mathrm{mM} \mathrm{MgCl}_{2}, 40 \mathrm{mM} \mathrm{NaCl}, 5 \%$ glycerol, $1 \mathrm{mM}$ dithiothreitol, and $0.2 \%$ Nonidet P-40) at $4{ }^{\circ} \mathrm{C}$. After lysis, samples were centrifuged for $2 \mathrm{~min}$ at 10,000 $\mathrm{Xg}$ to remove nuclei. The cytoplasmic extracts were diluted to a protein concentration of $3 \mathrm{mg} / \mathrm{mL}$ in lysis buffer without Nonidet P-40. The RNA IRE probe was transcribed in vitro from a linearized plasmid template (kindly provided by Dr. K. Pantopoulos) using T7 RNA polymerase in the presence of $\left[\alpha-{ }^{32} \mathrm{P}\right] \mathrm{UTP}$ as described [23]. For the RNA-protein complexes, $25 \mu \mathrm{g}$ of 


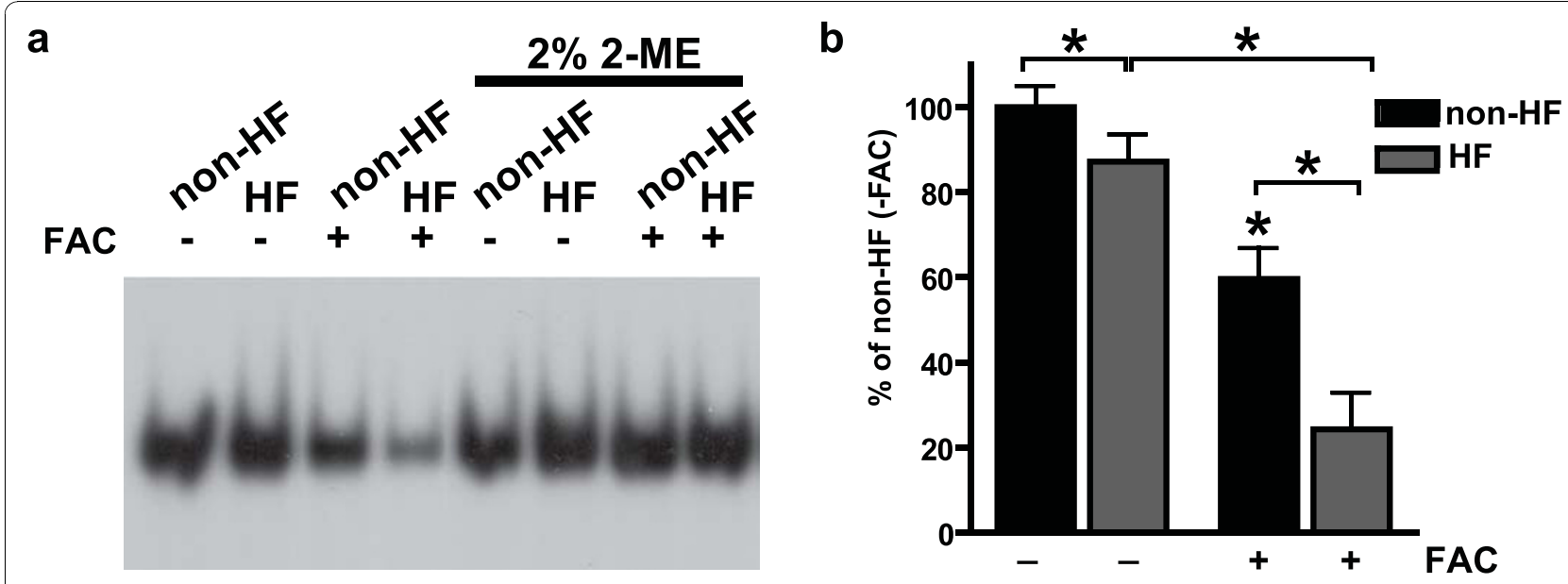

Figure 5 IRE-IRP binding activity is decreased in HF skin fibroblasts. a) Cytoplasmic extracts from non-HF and HF fibroblasts were analyzed for IRE-IRP binding activity using a gel-shift assay with a ${ }^{32}$ P-labelled RNA probe containing a ferritin IRE sequence in the absence or presence of 2\% 2-mercaptoethanol (2\% 2-ME) to promote maximal IRE-IRP1 binding. Fibroblasts were cultured in the presence (+) or absence $(-)$ of $100 \mu \mathrm{M}$ ferric ammonium citrate (FAC) for $72 \mathrm{~h}$. A lower binding activity of IRP1 was observed under basal conditions (no FAC) in HF fibroblasts. After FAC treatment, HF fibroblasts showed significantly less binding activity than controls (HF: $30 \pm 7 \%$; non-HF: $62 \pm 4 \%$ ). ${ }^{*} p<0.05$.

cytoplasmic extract was incubated at room temperature with the radiolabeled ferritin IRE probe $(25,000 \mathrm{cpm})$ in the absence or presence of 2\% 2-mercaptoethanol (2$\mathrm{ME})$. After $10 \mathrm{~min}$, heparin $(50 \mu \mathrm{g})$ was added to the reaction to inhibit non-specific protein interactions with

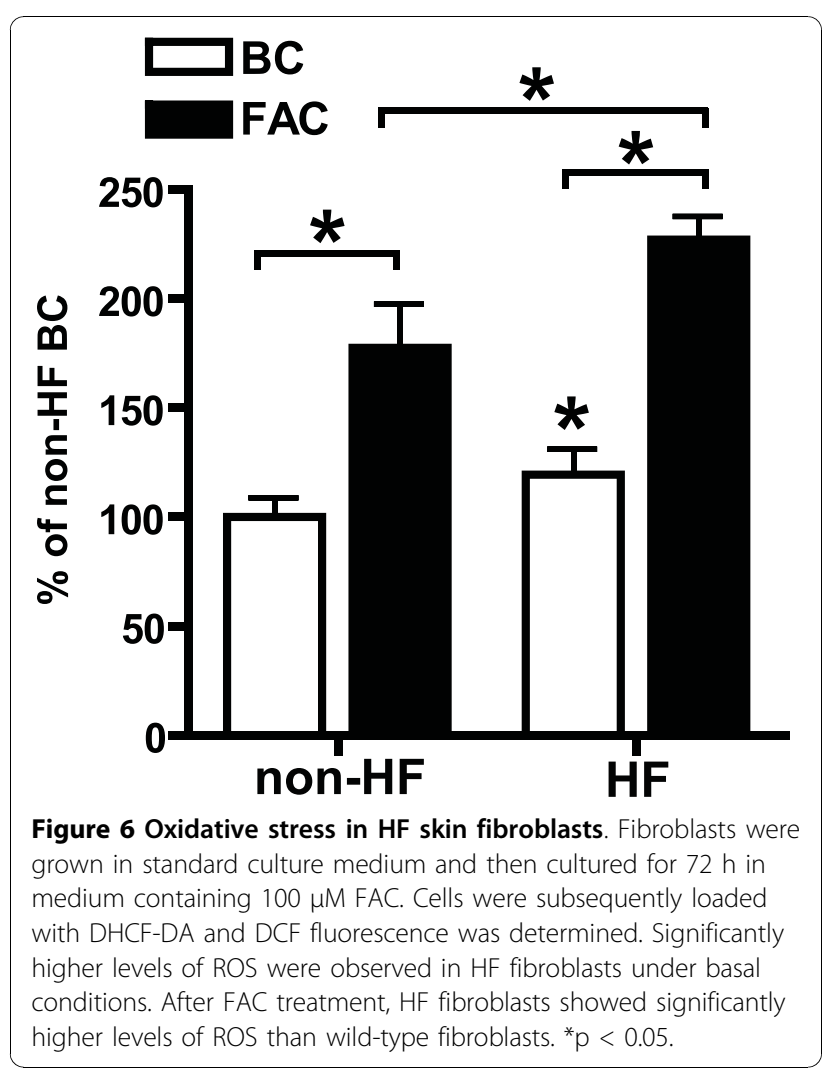

the probe and the incubation was continued for another $10 \mathrm{~min}$. Unprotected probe was degraded by incubation with $1 \mathrm{U}$ of RNase T1 for $10 \mathrm{~min}$. RNA-protein complexes were analyzed in $6 \%$ non-denaturing polyacrylamide gels and visualized by autoradiography. Bands were scanned and the ratios of the intensity of the IREIRP complexes formed in the absence or presence of 2-ME were determined.

\section{Quantitation of intracellular iron by the colorimetric ferrozine assay}

Total iron content was determined as previously described [18] with minor modifications. Briefly, cultured fibroblasts plated on $60 \mathrm{~mm}$ dishes were lysed in $0.9 \mathrm{ml}$ of $50 \mathrm{mM} \mathrm{NaOH}$ for $2 \mathrm{~h}$ on a shaker in a humidified atmosphere. A standard curve was set using FAC standards $(0-40 \mu \mathrm{M})$ in $10 \mathrm{mM} \mathrm{HCl}$. Aliquots of cell lysates or standards were placed in $1.5 \mathrm{~mL}$ tubes and mixed with $\mathrm{HCl}$ or $\mathrm{NaOH}$, respectively, to reach the same final concentration. Iron-releasing reagent (1.4M $\mathrm{HCl}: 4.5 \%(\mathrm{w} / \mathrm{v}) \mathrm{KMnO}_{4}$ in $\mathrm{H}_{2} \mathrm{O}$ ) was added and the mixture incubated for $2 \mathrm{~h}$ at $60^{\circ} \mathrm{C}$, in order to release iron-containing proteins. After cooling to room temperature, $60 \mu \mathrm{L}$ of the iron-detection reagent $(6.5 \mathrm{mM}$ ferrozine, $6.5 \mathrm{mM}$ neocuproine, $2.5 \mathrm{M}$ ammonium acetate, and $1 \mathrm{M}$ ascorbic acid dissolved in $\mathrm{H}_{2} \mathrm{O}$ ) was added to each tube. After $30 \mathrm{~min}, 280 \mu \mathrm{L}$ of the solution in each tube was transferred into a well of a 96-well plate and the absorbance was measured at $570 \mathrm{~nm}$ on a BioTek EL800 microplate reader (BioTek Instruments, Inc., Winooski, VT). The intracellular iron concentration was normalized against the protein content of the sample. 


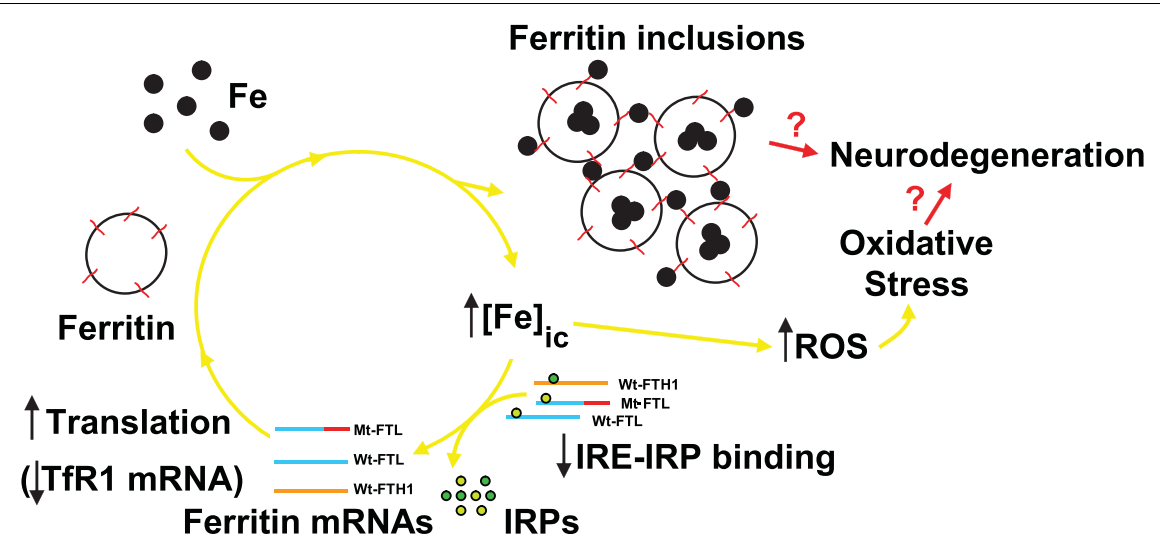

Figure 7 Iron-induced ferritin aggregation and oxidative stress in HF. The mutant FTL polypeptide acts as a dominant negative mutant, leading to a failure of ferritin in its iron storage function and an increase in the levels of intracellular (ic) iron, resulting in the release of the IRP proteins from the ferritin IRE and degradation of TfR1 MRNA, generating a positive feed-back loop that leads to over-production of ferritin polypeptides. The C-termini of the mutant FTL polypeptides may extend above the spherical shell allowing them to crosslink with other ferritin molecules through iron bridging, promoting iron-mediated aggregation of ferritin. Free iron leads to the generation of ROS and oxidative stress. Ferritin aggregates and oxidative stress may lead to neurodegeneration in HF.

\section{Determination of the Labile Iron Pool (LIP)}

LIP was measured using the metal-sensitive fluorescence probe calcein $[19,20]$ in attached cells as described [21] with minor modifications. Briefly, confluent cell monolayers were first treated for $72 \mathrm{~h}$ in the presence of vehicle or $100 \mu \mathrm{M}$ FAC. The cells were washed twice with PBS and loaded with calcein-acetoxymethyl ester (Invitrogen) at a final concentration of $0.25 \mu \mathrm{M}$ for $15 \mathrm{~min}$ at $37^{\circ} \mathrm{C}$ (from a $1 \mathrm{mM}$ stock solution in dimethyl sulfoxide) in loading medium (DMEM without sodium bicarbonate, $20 \mathrm{mM}$ Hepes pH 7.4, $1 \mathrm{mg} / \mathrm{ml}$ BSA). Cells were washed and loaded in measuring media (20 mM Hepes $\mathrm{pH} 7.4$, $150 \mathrm{mM} \mathrm{NaCl}, 5 \mathrm{mM}$ glucose). After reaching baseline (3 min) first fluorescence readings (F1) were taken using a Beckman DTX880 fluorimeter (Beckman Coulter Inc., Fullerton, CA) in bottom read technique with a fluorescein optical filter (excitation: $485 \mathrm{~nm}$; emission: $535 \mathrm{~nm}$ ). Finally, the cells were depleted of iron in the presence of the membrane-permeable iron chelator salicylaldehyde isonicotinoyl hydrazone ( $\mathrm{SIH})$, kindly provided by $\mathrm{Dr}$. Prem Ponka [40] and a second measurement of fluorescence was performed (F2) after $20 \mathrm{~min}$. The F2/F1 ratio was used as a relative indicator of LIP independent of the cell number and distribution within the wells.

\section{Quantification of reactive oxygen species (ROS)}

ROS formation was quantified by measuring fluorescence intensity of cultures after loading cells with 5-(and-6)chloromethyl-2',7'-dichlorodihydrofluorescein diacetate (CM- $\mathrm{H}_{2}$ DCFDA; Molecular Probes) [41]. CM- $\mathrm{H}_{2}$ DCFDA, a cell-permeable indicator for reactive oxygen species, is hydrolyzed inside cells to a non-fluorescent compound which emits fluorescence when oxidized. Cultures were washed with Hank's Balanced Salt Solution (HBSS) containing calcium and magnesium. $10 \mu \mathrm{M}$ DCFH-DA was loaded and incubated for $15 \mathrm{~min}$ at $37^{\circ} \mathrm{C}$. Cultures were then washed in HBSS and fluorescence measurements were taken at $37^{\circ} \mathrm{C}$ using a Beckman DTX880 fluorimeter in bottom read technique with a fluorescein optical filter (excitation: $485 \mathrm{~nm}$; emission: $535 \mathrm{~nm}$ ). Background fluorescence from cultures subjected to sham dye incubation was subtracted from each recording.

\section{Statistics}

All data are presented as the mean \pm SD. Samples were run on duplicate or triplicate and each experiment was repeated at least three times. Statistical analysis was performed using the Student's t-test or a one-way analysis of variance followed by the Student's-Newman-Keuls test for multiple comparisons using GraphPad Prism version 4.03 (GraphPad Software Inc, La Jolla, CA). Differences were declared statistically significant if $\mathrm{p}<0.05$. When normality test failed, comparison of the means was performed by Weltch test.

\section{Acknowledgements}

We are very grateful to all patients who through their commitment made this study possible. The authors are grateful to Virginie Garcia and Marie-Ange Gauthie-Berges for their technical assistance and Holly J. Garringer and Martin A Baraibar for helpful comments on the manuscript. We thank Dr. K. Pantopoulos for the pGEMI12.CAT plasmid and Dr. Prem Ponka for the iron chelator salicylaldehyde isonicotinoyl hydrazone. This study was supported by grants from the National Institute on Neurological Disorders and Stroke NS050227, NS063056 (RV) and the National Institute on Aging AG10133 (BG). The funding agencies had no influence over experimental design, data analysis or conclusions.

\section{Author details}

${ }^{1}$ Department of Pathology and Laboratory Medicine and Indiana Alzheimer disease Center, Indiana University School of Medicine, 635 Barnhill Dr, MSB A136, Indianapolis, IN, 46202, USA. Institut de Médecine Moléculaire de 
Rangueil, INSERM U.858, Université Toulouse III Paul-Sabatier, IFR31, Toulouse, France. ${ }^{3}$ Service d'Anatomie et de Cytologie Pathologiques, Hopitaux de Toulouse, TSA 50032 - 31059 Toulouse, Cedex 4, France.

\section{Authors' contributions}

AGB carried out experiments and analyzed data. TL and MBD extracted patients' fibroblasts and propagated them. BG critically read through the paper. AGB and RV designed the experiments and wrote the paper with assistance from all other authors. All authors read and approved the final manuscript.

\section{Competing interests}

The authors state that there is no actual or potential conflicts of interest including any financial, personal or other relationships with other people or organizations within five years of beginning the work submitted that could inappropriately influence (bias) their work. The authors do not have any non-financial competing interests (political, personal, religious, ideological, academic, intellectual, commercial or any other) to declare in relation to this manuscript.

Received: 12 August 2010 Accepted: 10 November 2010 Published: 10 November 2010

\section{References}

1. Beard JL, Connor JR: Iron status and neural functioning. Annu Rev Nutr 2003, 23:41-58.

2. Berg D, Youdim MB: Role of iron in neurodegenerative disorders. Top Magn Reson Imaging 2006, 17:5-17.

3. Vidal R, Delisle MB, Ghetti B: Neurodegeneration caused by proteins with an aberrant carboxyl-terminus. J Neuropathol Exp Neurol 2004, 63:787-800.

4. Curtis AR, Fey C, Morris CM, Bindoff LA, Ince PG, Chinnery PF, Coulthard A, Jackson MJ, Jackson AP, McHale DP, Hay D, Barker WA, Markham AF, Bates D, Curtis A, Burn J: Mutation in the gene encoding ferritin light polypeptide causes dominant adult-onset basal ganglia disease. Nat Genet 2001, 28:350-354.

5. Vidal R, Ghetti B, Takao M, Brefel-Courbon C, Uro-Coste E, Glazier BS, Siani V, Benson MD, Calvas P, Miravalle L, Rascol O, Delisle MB: Intracellular ferritin accumulation in neural and extraneural tissue characterizes a neurodegenerative disease associated with a mutation in the ferritin light polypeptide gene. J Neuropathol Exp Neurol 2004, 63:363-380.

6. Mancuso M, Davidzon G, Kurlan RM, Tawil R, Bonilla E, Di Mauro S, Powers JM: Hereditary ferritinopathy: a novel mutation, its cellular pathology, and pathogenetic insights. J Neuropathol Exp Neurol 2005, 64:280-294.

7. Ohta E, Nagasaka T, Shindo K, Toma S, Nagasaka K, Ohta K, Shiozawa Z: Neuroferritinopathy in a Japanese family with a duplication in the ferritin light chain gene. Neurology 2008, 70:1493-1494.

8. Devos D, Tchofo PJ, Vuillaume I, Destée A, Batey S, Burn J, Chinnery PF: Clinical features and natural history of neuroferritinopathy caused by the 458dupA FTL mutation. Brain 2009, 132:e109.

9. Kubota A, Hida A, Ichikawa Y, Momose Y, Goto J, Igeta Y, Hashida H, Yoshida K, Ikeda S, Kanazawa I, Tsuji S: A novel ferritin light chain gene mutation in a Japanese family with neuroferritinopathy: description of clinical features and implications for genotype-phenotype correlations. Mov Disord 2009, 24:441-445.

10. Ory-Magne F, Brefel-Courbon C, Payoux P, Debruxelles S, Sibon I, Goizet C, Labauge P, Menegon P, Uro-Coste E, Ghetti B, Delisle MB, Vidal R, Rascol O: Clinical phenotype and neuroimaging findings in a French family with hereditary ferritinopathy (FTL498-499InsTC). Mov Disord 2009, 24:1676-1683.

11. Theil EC: The ferritin family of iron storage proteins. Adv Enzymol Relat Areas Mol Biol 1990, 63:421-449.

12. Harrison PM, Arosio P: The ferritins: molecular properties, iron storage function and cellular regulation. Biochim Biophys Acta 1996, 1275:161-203.

13. Rucker $P$, Torti FM, Torti SV: Role of $H$ and $L$ subunits in mouse ferritin. $J$ Biol Chem 1996, 271:33352-33357.

14. Baraibar MA, Barbeito AG, Muhoberac BB, Vidal R: Iron-mediated aggregation and a localized structural change characterize ferritin from a mutant light chain polypeptide that causes neurodegeneration. I Biol Chem 2008, 283:31679-31689.
15. Baraibar MA, Muhoberac BB, Garringer HJ, Hurley TD, Vidal R: Unraveling of the E-helices and disruption of 4-fold pores are associated with iron mishandling in a mutant ferritin causing neurodegeneration. $J$ Biol Chem 2010, 285:1950-1956

16. Vidal R, Miravalle L, Gao X, Barbeito AG, Baraibar MA, Hekmatyar SK, Widel M, Bansal N, Delisle MB, Ghetti B: Expression of a mutant form of the ferritin light chain gene induces neurodegeneration and iron overload in transgenic mice. J Neurosci 2008, 28:60-67.

17. Barbeito AG, Garringer HJ, Baraibar MA, Gao X, Arredondo M, Núñez MT, Smith MA, Ghetti B, Vidal R: Abnormal iron metabolism and oxidative stress in mice expressing a mutant form of the ferritin light polypeptide gene. J Neurochem 2009, 109:1067-1078.

18. Riemer J, Hoepken HH, Czerwinska H, Robinson SR, Dringen R: Colorimetric ferrozine-based assay for the quantitation of iron in cultured cells. Anal Biochem 2004, 331:370-375.

19. Breuer W, Epsztejn S, Millgram P, Cabantchik IZ: Transport of iron and other transition metals into cells as revealed by a fluorescent probe. Am J Physiol 1995, 268:1354-1361.

20. Breuer W, Epsztejn S, Cabantchik ZI: Iron acquired from transferrin by K562 cells is delivered into a cytoplasmic pool of chelatable iron(II). $J$ Biol Chem 1995, 270:24209-24215.

21. Andriopoulos B, Hegedusch S, Mangin J, Riedel HD, Hebling U, Wang J, Pantopoulos K, Mueller S: Sustained hydrogen peroxide induces iron uptake by transferrin receptor- 1 independent of the iron regulatory protein/iron-responsive element network. J Biol Chem 2007, 282:20301-20308

22. Kim S, Ponka P: Control of Transferrin Receptor Expression via Nitric Oxide-mediated Modulation of Iron-regulatory Protein 2. J Biol Chem 1999, 274:33035-33042

23. Mueller S, Pantopoulos K: Activation of iron regulatory protein-1 (IRP1) by oxidative stress. Methods Enzymol 2002, 348:324-337.

24. Vidal R, Delisle MB, Rascol O, Ghetti B: Hereditary ferritinopathies. In Neurodegeneration. The Molecular Pathology of Dementia and Movement Disorders. 2 edition. Edited by: Dickson D, Weller R. Wiley-Blackwell;

25. Deng $X$, Vidal R, Englander EW: Accumulation of oxidative DNA damage in brain mitochondria in mouse model of hereditary ferritinopathy. Neurosci Lett 2010, 479:44-48.

26. Alkhateeb AA, Connor JR: Nuclear ferritin: A new role for ferritin in cell biology. Biochim Biophys Acta 2010, 1800(8):793-7.

27. Smith AG, Carthew P, Francis JE, Edwards RE, Dinsdale D: Characterization and accumulation of ferritin in hepatocyte nuclei of mice with iron overload. Hepatology 1990, 12:1399-1405.

28. Linsenmayer TF, Cai CX, Millholland JM, Beazley KE, Fitch JM: Nuclear ferritin in corneal epithelial cells: tissue-specific nuclear transport and protection from UV-damage. Prog Retin Eye Res 2005, 24:139-159.

29. Thompson KJ, Fried MG, Ye Z, Boyer P, Connor JR: Regulation, mechanisms and proposed function of ferritin translocation to cell nuclei. J Cell Sci 2002, 115:2165-2177.

30. Muhoberac BB, Baraibar MA, Vidal R: Iron loading-induced aggregation and reduction of iron incorporation in heteropolymeric ferritin containing a mutant light chain that causes neurodegeneration. Biochim Biophys Acta.

31. Hentze MW, Muckenthaler MU, Andrews NC: Balancing acts: molecular control of mammalian iron metabolism. Cell 2004, 117:285-297.

32. Hentze MW, Kühn LC: Molecular control of vertebrate iron metabolism: mRNA-based regulatory circuits operated by iron, nitric oxide, and oxidative stress. Proc Natl Acad Sci USA 1996, 93:8175-8182.

33. Garrick LM, Dolan KG, Romano MA, Garrick MD: Non-transferrin-bound iron uptake in Belgrade and normal rat erythroid cells. I Cell Physiol 1999, 178:349-358.

34. Salazar J, Mena N, Hunot S, Prigent A, Alvarez-Fischer D, Arredondo M, Duyckaerts C, Sazdovitch V, Zhao L, Garrick LM, Nuñez MT, Garrick MD, Raisman-Vozari R, Hirsch EC: Divalent metal transporter 1 (DMT1) contributes to neurodegeneration in animal models of Parkinson's disease. Proc Natl Acad Sci USA 2008, 105:18578-83.

35. Kakhlon O, Cabantchik Zl: The labile iron pool: characterization, measurement, and participation in cellular processes. Free Radic Biol Med 2002, 33:1037-1046. 
36. Cozzi A, Rovelli E, Frizzale G, Campanella A, Amendola M, Arosio P, Levi S: Oxidative stress and cell death in cells expressing L-ferritin variants causing neuroferritinopathy. Neurobiol Dis 2010, 37:77-85.

37. Zecca L, Youdim MB, Riederer P, Connor JR, Crichton RR: Iron, brain ageing and neurodegenerative disorders. Nat Rev Neurosci 2004, 5:863-873.

38. Ke Y, Qian ZM: Brain iron metabolism: neurobiology and neurochemistry. Prog Neurobiol 2007, 83:149-173.

39. Sayre LM, Perry G, Smith MA: Redox metals and neurodegenerative disease. Curr Opin Chem Biol 1999, 3:220-225.

40. Laskey J, Webb I, Schulman HM, Ponka P: Evidence that transferrin supports cell proliferation by supplying iron for DNA synthesis. Exp Cell Res 1988, 176:87-95.

41. Reynolds IJ, Hastings T: Glutamate induces the production of reactive oxygen species in cultured forebrain neurons following NMDA receptor activation. J Neurosci 1995, 15:3318-3327.

doi:10.1186/1750-1326-5-50

Cite this article as: Barbeito et al:: Abnormal iron metabolism in fibroblasts from a patient with the neurodegenerative disease hereditary ferritinopathy. Molecular Neurodegeneration 2010 5:50.

\section{Submit your next manuscript to BioMed Central} and take full advantage of:

- Convenient online submission

- Thorough peer review

- No space constraints or color figure charges

- Immediate publication on acceptance

- Inclusion in PubMed, CAS, Scopus and Google Scholar

- Research which is freely available for redistribution

Submit your manuscript at www.biomedcentral.com/submit 